\title{
Mehr Engagement der Politikwissenschaft in der Anthropozän-Debatte
}

\author{
Thomas Hickmann (D) - Lena Partzsch • Philipp Pattberg • \\ Sabine Weiland
}

Online publiziert: 2 . November 2020

(C) Der/die Autor(en) 2020

Zusammenfassung Die aktuelle Debatte um das Anthropozän wird bislang von den Naturwissenschaften dominiert. Dies führt zu einer verkürzten Analyse von Naturzerstörungen und der zugrundliegenden Veränderungen der Mensch-UmweltBeziehungen. In diesem Kommentar fordern wir die Politikwissenschaft auf, sich an der Debatte über das sogenannte Menschenzeitalter und seine weitreichenden Implikationen stärker zu beteiligen. Das Anthropozän zeichnet sich insbesondere durch komplexe Wechselwirkungen, nicht-lineare Dynamiken und Kipppunkte aus, an denen Entwicklungen umschlagen. Vor diesem Hintergrund wird deutlich, dass es im Anthropozän kein einfaches Management des menschlichen Einflusses auf das Erdsystem geben kann. Um die Ursachen und potenziellen Lösungen menschengemachter Umweltprobleme zu erforschen, sind Fragen nach Machtverhältnissen, Interessengegensätzen sowie Normkonflikten zentral. Die Politische Theorie ist zudem für die Beantwortung der Frage, wie sich die existierenden politischen Institutionen im Anthropozän reformieren lassen, höchst relevant. Der Politikwissenschaft kommt daher in der Anthropozän-Debatte eine besondere Rolle zu. Das Anthropozän sollte dabei nicht nur ein Thema für die umweltpolitikwissenschaftliche Forschung blei-

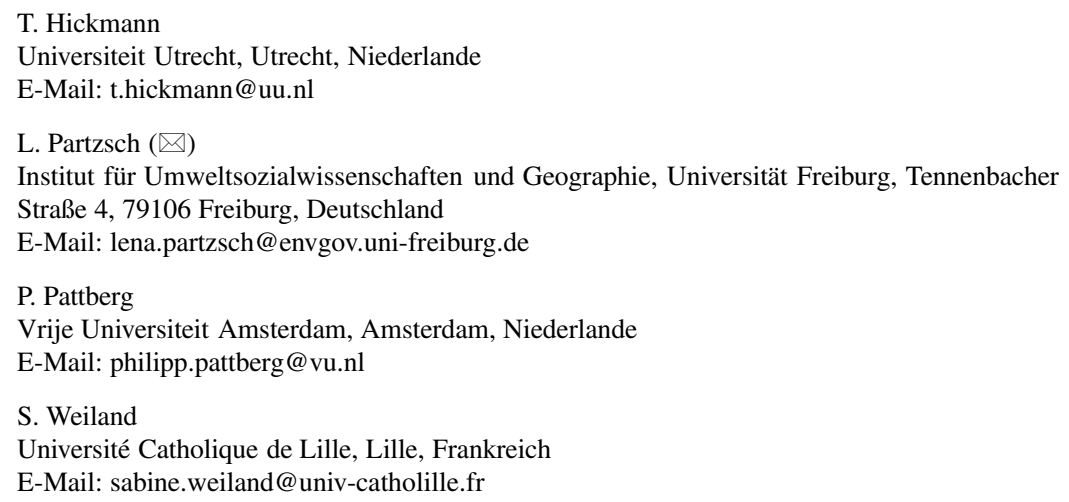


ben. Vielmehr ist ein Beitrag der Politikwissenschaft in der gesamten Breite der Disziplin notwendig, um in einem interdisziplinären Dialog adäquate Antwortstrategien auf systemgefährdende globale Umweltveränderungen zu entwickeln.

Schlüsselwörter Anthropozän · Governance · Interdisziplinarität · Politische Theorie $\cdot$ Sozial-ökologische Transformation

\title{
More Engagement of Political Science in the Anthropocene Debate
}

\begin{abstract}
The current debate on the Anthropocene is thus far dominated by the natural sciences. This leads to a simplistic analysis of environmental devastation and the underlying changes of human-nature relations. In this commentary, we urge the discipline of political science to enhance its engagement in the debate on the so-called human age and its far-reaching implications. The Anthropocene is characterized first and foremost by complex interactions, non-linear dynamics and tipping points that entail abrupt changes. Against this backdrop, it becomes clear that there can be no simple management of human impacts on the earth system in the Anthropocene. To explore the root causes and potential solutions of human-induced environmental problems, we need to raise questions on key topics, such as prevailing power relations, clashes of interests and norm conflicts. Political theory is moreover highly relevant to address the question of how the existing political institutions could be reformed in the Anthropocene. The discipline of political science plays hence a particular role in the Anthropocene debate. Yet, the Anthropocene should not only remain a critical topic for those political scientists concerned with the environment. Instead, what is needed is a political science contribution that reflects the entire breadth of the discipline to develop adequate response strategies for system-threatening global environmental changes in an interdisciplinary dialogue.
\end{abstract}

Keywords Anthropocene - Governance - Interdisciplinarity · Political theory · Social-ecological transformation

\section{Einleitung}

Zahlreiche Studien der letzten Jahre belegen, dass der Eingriff des Menschen in das Erdsystem ein neues Ausmaß erreicht hat. Vor diesem Hintergrund haben der Chemie-Nobelpreisträger Paul Crutzen und der Biologe Eugene Stoermer (2000) vorgeschlagen, eine neue Epoche in der Erdgeschichte auszurufen: das Anthropozän. Der Begriff drückt aus, dass der Mensch zur treibenden Kraft natürlicher Stoffkreisläufe und Prozesse auf der Erde geworden ist. Das Anthropozän löst damit das Holozän nach etwa 12.000 Jahren als gegenwärtige erdgeschichtliche Epoche ab. Die durch den Menschen herbeigeführten und dramatisch beschleunigten Umweltveränderungen wie Klimawandel, Artensterben und Bodendegradation bergen die Gefahr eines irreversiblen Systemwandels, der die Bewohnbarkeit der Erde für viele Lebewesen potenziell langfristig einschränkt (Steffen et al. 2018). 
Die Politikwissenschaft hat zu der mittlerweile 20-jährigen Debatte um das Anthropozän bislang wenig beigetragen. Erst in den letzten Jahren wurden einige politikwissenschaftliche Perspektiven auf das Anthropozän entworfen und in die Diskussion eingebracht (z.B. Pattberg und Zelli 2016; Dryzek und Pickering 2018; Biermann und Lövbrand 2019; Hickmann et al. 2019; Rothe 2020). Für die umweltpolitikwissenschaftliche Forschung ergibt sich mit der Ausrufung des Anthropozän die Herausforderung, Mensch-Umwelt-Beziehungen nach dem proklamierten „Ende der Natur“ (McKibben 1990) neu zu denken. Laut Christoph Görg tritt im Anthropozän die „Dialektik der Naturbeherrschung“ in eine neue Phase (2016, S. 11). In der Folge wird nicht nur das Verhältnis von Natur und Gesellschaft gründlich durcheinandergewirbelt, sondern auch ,die Grundlagen politischer Entscheidungen und demokratischer Prozeduren, die weder zu den geologischen Zeitskalen noch zur Dringlichkeit einer Entschleunigung des Ressourcenverbrauchs passen" (Görg 2016, S. 13). Dies sind genuin politikwissenschaftliche Fragen, denen sich unsere Disziplin stellen sollte.

Wir knüpfen an diese Beobachtung an und fordern ein stärkeres politikwissenschaftliches Engagement mit dem ausgerufenen Zeitalter des Menschen. Jenseits der laufenden naturwissenschaftlichen Definitions- und Datierungsversuche kommt der Politikwissenschaft die Aufgabe zu, die weitreichenden politischen und gesellschaftlichen Implikationen des Anthropozän-Konzepts herauszuarbeiten. Sie wurden bisher in der naturwissenschaftlich dominierten Debatte weitgehend ausgeblendet, sind jedoch entscheidend zur Richtungsbestimmung bei der gegenwärtigen Suche nach einer umfassenden sozial-ökologischen Transformation. Dabei reicht es nicht aus, das Anthropozän lediglich als umweltpolitikwissenschaftliches Thema zu begreifen, etwa im Sinne einer Politikfeldanalyse. Vielmehr bedarf es der Auseinandersetzung mit dem Thema in der gesamten Breite der Politikwissenschaft.

In diesem Kommentarbeitrag konzentrieren wir uns nach einem kurzen Abriss zum Hintergrund des Anthropozän-Begriffs auf drei Aspekte: Erstens argumentieren wir mit Blick auf das Governance-Konzept, dass im Anthropozän kein einfaches Management des menschlichen Einflusses auf das Erdsystem möglich ist. Vielmehr sind Fragen nach Machtverhältnissen, Interessengegensätzen sowie Normkonflikten zentral, um Ursachen menschengemachter Umweltveränderungen zu erforschen. Zweitens unterstreichen wir die Relevanz der Politischen Theorie für die Reflexion des sich wandelnden Mensch-Natur-Verhältnisses sowie zur Beantwortung der Frage, wie sich die existierenden politischen Institutionen im Anthropozän sinnvoll reformieren lassen. Und drittens plädieren wir für eine umfassende Einmischung der Politikwissenschaft in eine interdisziplinäre Anthropozän-Debatte mit dem Ziel, die politische Dimension des Menschenzeitalters herauszuarbeiten. In Kombination mit anderen Disziplinen können daraus Antwortstrategien auf zunehmende Erdsystemveränderungen und die damit einhergehenden dramatischen Folgewirkungen entwickelt werden. 


\section{Hintergrund des Anthropozän-Begriffs}

Die Anthropozän-These besagt im Kern, dass der Mensch als eine neue und einflussreiche Naturkraft im System Erde hervortritt und damit zum geologischen Faktor und entscheidenden Treiber für globale Wandlungsprozesse wird. Dies wird als ein in der Erdgeschichte einmaliges Ereignis beschrieben: „For the first time a single species dominates the entire surface, sits at the top of all terrestrial and oceanic food chains, and has taken much of the biosphere for its own purposes" (Langmuir und Broecker 2012, S. 597). Es stellt zugleich eine fundamentale Veränderung in den Mensch-Umwelt-Beziehungen dar, in dessen Zentrum nun die Dominanz des Menschen über biologische, chemische und damit letztlich geologische Prozesse auf der Erde steht. Paul Crutzen und Christian Schwägerl (2011) formulieren es folgendermaßen: „It's no longer us against ,Nature'. It's we who decide what nature is and what it will be".

Dieser Befund unterstreicht die erheblichen politischen und gesellschaftlichen Implikationen des Anthropozän-Begriffs. Zuallererst offenbart er die Dringlichkeit der Bekämpfung globaler Umweltprobleme. Wie sich eindrücklich an der Klimapolitik zeigt, ist es in den letzten drei Jahrzehnten trotz wachsender Gewissheit über die Ursachen des Klimawandels nicht gelungen, entsprechende Maßnahmen gegen die vom Menschen bewirkte erhöhte atmosphärische Konzentration von Treibhausgasen einzuleiten. Es ist unklar, ob und wie es gelingt, die Erderwärmung auf $1,5^{\circ} \mathrm{C}$ gegenüber dem vorindustriellen Niveau zu begrenzen (Rockström et al. 2016). Zwar haben sich im Pariser Klimaabkommen vom Dezember 2015 praktisch alle Staaten zu eigenen Schritten zur Senkung oder Begrenzung ihrer Treibhausgasemissionen verpflichtet. Die USA und Brasilien treiben jedoch ihren Ausstieg aus dem Abkommen voran und auch in anderen Ländern mangelt es an den notwendigen Ambitionen oder einer konkreten Umsetzung der selbst gesteckten Ziele. In diesem Kontext kann das Anthropozän als Drohkulisse und Handlungsaufforderung verstanden werden, insbesondere mit Blick auf sogenannte Kipp-Punkte und die damit einhergehenden abrupten Veränderungen und Rückkopplungseffekte, die das gesamte Erdsystem aus dem Gleichgewicht bringen können und damit die menschliche Lebens- und Entwicklungsgrundlage gefährden (Lenton 2011).

Darüber hinaus verändert der Begriff des Anthropozän auch die Sichtweise auf die Regulierbarkeit globaler Umweltveränderungen. Eine Reihe von Autorinnen und Autoren stellen dabei die Verantwortung (im englischen Sprachgebrauch als ,stewardship" bezeichnet) der Menschheit heraus, die Erde als Lebensgrundlage für heutige und zukünftige Generationen zu erhalten (z. B. Steffen et al. 2011). Ihnen zufolge liegt es in unseren Händen, wie wir das Menschenzeitalter gestalten und ob es in eine Katastrophe führt oder nicht. Die Autorinnen und Autoren fordern daher entsprechende Reformen in allen gesellschaftlichen und politischen Bereichen. Gleichzeitig gibt es in der Debatte auch Tendenzen, die Bedingungen des Anthropozän als gegeben hinzunehmen und auf globale Umweltveränderungen lediglich zu reagieren (Stirling 2014). Ein Diskussionsstrang befasst sich speziell mit den Möglichkeiten, die diagnostizierten Systemstörungen in den Zustand eines Good Anthropocene zu überführen (z.B. Bosselmann 2015). So betonen einige Expertinnen und Experten, dass der Mensch mithilfe technologischer Innovationen bei- 
spielsweise eine effizientere Landwirtschaft betreiben oder durch Geo-Engineering den anthropogenen Treibhauseffekt kontrollieren könne. In diesen Argumentationen ist die Idee zentral, dass die Ansätze und Ideen für eine Kontrolle oder positive Beeinflussung des Anthropozän bereits gesät seien und nun ihre praktische Anwendung beginne (Bennett et al. 2016).

Andere Wissenschaftlerinnen und Wissenschaftler kritisieren dagegen, dass das Anthropozän-Konzept die Menschheit als eine aus der Natur herausgehobene Art betone. Dadurch werde der ungehemmten Umgestaltung der Erde durch den Menschen weiter Vorschub geleistet (Manemann 2014). Insbesondere werde die Beeinflussbarkeit von Naturkreisläufen durch den Menschen zum Anlass genommen und als Rechtfertigung gebraucht, um mit dem Anspruch der Reparatur gezielt auf ökologische Zusammenhänge und Prozesse einzuwirken (Vaughan und Lenton 2011). Vor Visionen und Modellen eines planetarischen Managements des Erdsystems, die auf hierarchischen und autoritativen Steuerungsvorstellungen basieren und den Prinzipien liberaler demokratischer Gesellschaften entgegenstehen, wird gewarnt (Eckersley 2015; Blühdorn 2020). In diesem Kontext heben Forscherinnen und Forscher des Earth-System-Governance-Projekts die komplexe und unvermeidlich politische Dimension von Governance auf allen politisch-administrativen Ebenen hervor und stehen damit simplifizierenden Management- und Kontrollvorstellungen kritisch gegenüber (Biermann 2014).

Die Politikwissenschaft kann Antworten auf die genannten Fragen geben und damit Dimensionen erschließen, die bisher in der Anthropozän-Debatte vernachlässigt werden. Im Folgenden arbeiten wir den genuinen Beitrag der Politikwissenschaft zur Debatte über die gravierenden Implikationen des Anthropozän-Konzepts anhand einer Reihe einschlägiger Beispiele heraus. Wir beginnen mit einer Diskussion zur Komplexität von Governance im Anthropozän und zur Relevanz von Macht- und Verteilungsfragen. Danach betonen wir die Bedeutung der Politischen Theorie für gesellschaftliche und politische Reformprozesse im Anthropozän. Dies führt uns schließlich zu einem Plädoyer für ein stärkeres Engagement der Politikwissenschaft in einer interdisziplinären Debatte darüber, wie nachhaltige Strategien zur Bewältigung und Vermeidung globaler Umweltprobleme formuliert werden können.

\section{Governance im Anthropozän}

Nimmt man die Bedeutung des Anthropozän-Begriffs ernst, besteht für alle Akteure auf den verschiedenen Ebenen des politisch-administrativen Systems ein weitreichender und dringender Handlungsbedarf. Biermann et al. formulieren es in Anbetracht der Anthropozän-These wie folgt: ,Societies must change course to steer away from critical tipping points in the Earth system that might lead to rapid and irreversible change. This requires fundamental reorientation and restructuring of national and international institutions toward more effective Earth system governance and planetary stewardship“ (2012, S. 1306). Erkenntnisse politikwissenschaftlicher Governance-Forschung sind daher grundsätzlich sehr gefragt. Dabei wird jedoch in vielen Wissenschaftskreisen von einem weitgehend rationalen Modell der Entscheidungsfindung ausgegangen. Aus unserer Sicht ist es von zentraler Bedeutung, weit 
verbreitete technische und unpolitische Auffassungen von Governance als einfachem Management des menschlichen Einflusses auf die Naturkreisläufe zu ersetzen durch ein tiefergehendes Verständnis politischer Steuerungsprozesse, die mit Konflikten und Aushandlungsprozessen verbunden sind. Insbesondere sollten in der Anthropozän-Debatte Aspekte des Governance-Konzepts mehr Beachtung finden, die sich mit Macht- und Gerechtigkeitsfragen, Komplexität und Steuerbarkeit sowie der Frage nach nichtintendierten Effekten auseinandersetzen. Die Politikwissenschaft kann in ihrer ganzen Breite dazu entscheidende Beiträge liefern.

In erster Linie erfordert Governance im Anthropozän politische Aktivitäten und Aushandlungen, die auf norm- und wertebasierten Entscheidungen beruhen und typischerweise Verteilungseffekte nach sich ziehen. Das bekannte Diktum „Wer bekommt was, wann, wie?“ (Lasswell 1936) kann in diesem Kontext als Leitfaden dienen, um die Widersprüche und Auseinandersetzungen über verschiedene Governance-Instrumente zu analysieren. Viele Autorinnen und Autoren haben diese Themen mit Fokus auf einzelne Politikfelder untersucht (z. B. Okereke 2010; Kalfagianni und Skordili 2018). In der Anthropozän-Debatte bleiben Gerechtigkeitsfragen jedoch bislang im Hintergrund. Dabei sind sie elementar, sowohl für die Erforschung der gesellschaftlichen Konsequenzen globaler Umweltveränderungen als auch für die Formulierung von adäquaten und gesellschaftlich akzeptierten Gegenmaßnahmen. Zudem ist die genaue Definition und Datierung des Anthropozän selbst politisch. So zeigten Philipp Pattberg und Michael Davis-Venn kürzlich, welche impliziten Annahmen über politische Steuerung in verschiedenen Datierungsvorschlägen zum Anthropozän versteckt sind. Ihr Fazit lautet: ,,[D]eciding on an appropriate start date and related marker is more than a technical-administrative act. In this decision are embedded potentially different narratives of the Anthropocene, which, depending on the choices made, will shape future governance and societal debates" (2020, S. 130).

Darüber hinaus ist Governance weitaus komplexer als vielfach in naturwissenschaftlichen Kreisen angenommen. Bislang hat die Anthropozän-Debatte vor allem gezeigt, wie vertrackt und in sich verwoben die verschiedenen Erdsystemkomponenten sind (Reid et al. 2010). Diese Erkenntnisse sind fundamental, um die natürlichen Zusammenhänge im Erdsystem zu verstehen und Entscheidungsgrundlagen für politische Maßnahmen zu erarbeiten. Ebenso komplex sind jedoch auch die existierenden Governance-Ansätze im Mehrebenensystem (Berkes 2017; Pattberg und Widerberg 2019). Eine Vielzahl an Akteuren mit unterschiedlichen Interessen und Motiven ist an der Schaffung formeller und informeller Regelsysteme im Anthropozän beteiligt. Die politikwissenschaftliche Forschung hat in den letzten Jahren mit der Entwicklung des Konzepts des Regimekomplexes und der institutionellen Fragmentierung dazu beigetragen, Governance-Komplexität im Anthropozän abzubilden (Biermann et al. 2009; Keohane und Victor 2011; Orsini et al. 2019). Aus dieser Komplexität heraus ergeben sich viele nichtlineare Entwicklungen, die nur schwer vorhersehbar sind. Rationale Planung ist daher nur in sehr seltenen Fällen und in begrenztem Ausmaß möglich. Politikwissenschaftliche Erkenntnisse zu Governance im Mehrebenensystem sowie den Grenzen der Rationalität im Risikozeitalter können hier entscheidende Einsichten liefern.

Darüber hinaus ist Governance immer mit unsicherem Ausgang verbunden (Lövbrand et al. 2009). Selbst wenn Governance-Instrumente gut designt und umgesetzt 
werden, sind ihre Wirkungen vielfach nicht eindeutig nachzuweisen oder erst mit längerem Abstand sinnvoll zu evaluieren. Neben der Möglichkeit der nichtintendierten Effekte werden Governance-Instrumente immer wieder neu verhandelt, von verschiedenen Interessengruppen infrage gestellt und dadurch verändert. Folglich können sie ihr Ziel verfehlen. Wichtig ist es daher, die Möglichkeit des Scheiterns von Governance-Initiativen einzuräumen. Gleichzeitig zeigen aktuelle Ansätze zu Governance-Experimenten (Hoffmann 2011; Overdevest und Zeitlin 2014; Kivimaa et al. 2017), wie erfolgreiche Experimente durch Diffusionsprozesse eine Breitenwirkung entfalten und somit zu Bausteinen im politischen Gestaltungsprozess des Anthropozän werden können.

\section{Politische Theorie im Anthropozän}

Im Mittelpunkt der Anthropozän-Debatte steht das Mensch-Natur-Verhältnis - ein Thema, mit dem sich die Politische Theorie seit ihren Ursprüngen beschäftigt, angefangen bei Platon in der Antike, über Immanuel Kant in der Aufklärung bis hin zu feministischen Auseinandersetzungen mit modernen Naturverhältnissen (Dobson 2007). Diese theoretischen Abhandlungen können dabei helfen, das Verhältnis zwischen Mensch und Natur vor dem Hintergrund des zunehmenden menschlichen Einflusses auf die Umwelt neu zu definieren, ohne in alte Muster der Naturbeherrschung zu verfallen. Durch den Menschen verursachte oder beschleunigte, aber eben nicht naturgegebene Veränderungen sind eine Folge gesellschaftlichen und politischen Handelns (Jamieson und Di Paola 2016). Zugleich ist die Menschheit essenzieller Bestandteil der Evolution und damit der Natur. Das Anthropozän verweist insofern auf die Unausweichlichkeit von Politik, um auf Grundlage von Aushandlungsprozessen ein neues Verständnis vom Mensch-Natur-Verhältnis zu entwickeln. Eine „RePolitisierung“ des Anthropozän eröffnet somit eine Debatte über globale Umweltprobleme, die über die bisherigen ontologisierenden Ansätze hinausgeht (Biermann und Lövbrand 2019). Diese muss im Kern - Stichwort: Post-Politik - und trotz ihrer teilweise apokalyptischen Rhetorik über die pragmatische Verteidigung des Status quo hinausgehen.

Das Ziel politikwissenschaftlicher Forschung in Bezug auf das Anthropozän sollte es sein, diverse Perspektiven, Problembeschreibungen und Zukunftsideen über die Gestalt von Umwelt- und Nachhaltigkeitspolitik herauszuarbeiten. Auf dieser Basis können Räume zur politischen Diskussion mit zivilgesellschaftlicher Partizipation geschaffen werden. Solche Räume böten die Chance, im Anthropozän eben nicht das „Ende der Politik“ zu sehen, sondern das genaue Gegenteil: eine Epoche, in der die Menschheit einen bewussten und nachhaltigen Umgang mit ihrer Umwelt pflegt und gestaltet. $\mathrm{Zu}$ einer ähnlichen Schlussfolgerung kommen Eva Lövbrand et al.: „We believe that a deeper involvement of critical social science in global environmental change research represents an important step out of this post-political situation“" (2015, S. 214).

Darüber hinaus wird im Licht der Anthropozän-These die kosmopolitische Perspektive geschärft (Alcaraz et al. 2016). Ethische Konzepte konzentrieren sich in der klassischen politischen Theorie und teilweise bis heute auf Beziehungen zwischen 
benachbarten Gemeinschaften und Staaten. Menschen auf der anderen Seite des Erdballs sind dabei nicht Teil ethischer Überlegungen und Prinzipien, ganz zu schweigen von zukünftigen Generationen und ihren Rechten. Dies erklärt zumindest teilweise, warum es leichter ist, für lokale Umweltschutzmaßnahmen zu mobilisieren statt für globalen Klimaschutz. Das Anthropozän fordert diese Denkweise jedoch heraus und provoziert einen Diskurs über eine umfassendere globale Ethik (Schmidt et al. 2016). Zwar sind nicht alle Menschen gleichermaßen von Umweltveränderungen betroffen, aber letztlich sitzt die Menschheit in einem Boot, da im Fall eines irreversiblen Systemwandels die Lebensgrundlagen für alle Menschen zerstört werden. Nur durch neue Formen des grenzüberschreitenden Austauschs kann es gelingen, die gegenseitigen Abhängigkeiten und die damit einhergehende Verwundbarkeit der Menschheit zu begreifen (Chandler und Reid 2016). Auf dieser Grundlage lassen sich neue Formen globaler Kooperation schaffen, um aktuelle und zukünftige globale Herausforderungen zu bewältigen.

In praktischer Hinsicht kann die Politische Theorie somit wichtige Antworten auf die Frage geben, wie die existierenden politischen Institutionen im Anthropozän reformiert werden können. Ein konkretes Beispiel ist die Forderung nach einer Einrichtung von politischen Gremien auf allen politischen Ebenen, in denen die Interessen der Jugend und zukünftiger Generationen repräsentiert werden (Tremmel 2015). In solchen Zukunftsgremien könnten die Rechte nachfolgender Generationen diskutiert und verteidigt werden und auf diese Weise ein wichtiger Anstoß zu den aktuellen Reformbemühungen des bestehenden institutionellen Systems hin zu mehr Nachhaltigkeit geliefert werden (González-Ricoy und Gosseries 2016). Ein solches verstärktes Bewusstsein für eine generationenübergreifende Ethik und Gerechtigkeit kann dazu beitragen, die Menschheit nicht (mehr) als von der Natur losgelöst zu sehen, sondern sie stattdessen als ein sich wandelnder Teil von ihr zu verstehen.

\section{Die Rolle der Politikwissenschaft in der interdisziplinären Anthropozän-Debatte}

Die Debatte um das Anthropozän wird trotz der entworfenen politikwissenschaftlichen Arbeiten weiter von den Naturwissenschaften dominiert. Wir richten deshalb einen Appell und eine Einladung an unsere eigene Disziplin, sich konstruktiv und selbstbewusst in die Debatte einzubringen. Der Politikwissenschaft kommt dabei wie gezeigt eine zentrale Rolle als Richtungsgeberin auf der Suche nach geeigneten und gesellschaftlich anerkannten Wegen zu einer umfassenden sozial-ökologischen Transformation zu.

Die wegweisende Rolle der Politikwissenschaft in der Anthropozän-Debatte herauszustellen, bedeutet indes nicht, dass die Debatte nur oder federführend hier geführt werden sollte. Es geht nicht darum, einzelne Wissenschaftsdisziplinen gegeneinander auszuspielen oder eine Hierarchie zwischen ihnen zu begründen. Im Mittelpunkt der Anthropozän-Debatte steht die enorme Herausforderung, die negativen Auswirkungen des menschlichen Einflusses auf die natürlichen Lebensgrundlagen des Planeten zu verhindern. Hier wird die Notwendigkeit eines wechselseitigen Austauschs zwischen den verschiedenen Disziplinen deutlich. Ohne stabile Brücken 
zwischen den Fächern bleibt jede Disziplin für sich alleine ratlos, wenn es darum geht, sinnvolle Lösungsansätze für eine nachhaltigere Wirtschaftsweise und Entwicklung zu liefern (Frodeman et al. 2017).

Aufgrund der starken Ausdifferenzierungen und fachlichen Spezialisierungen sowie eigener Terminologien in den verschiedenen wissenschaftlichen Disziplinen ist eine fächerübergreifende Zusammenarbeit anspruchsvoll. Davon zeugen viele interdisziplinäre Forschungsprojekte, in denen zunächst mühsam eine gemeinsame Sprache als Basis für ein gegenseitiges Verständnis gefunden werden muss. Das Anthropozän kann in diesem Kontext als Antrieb und als Brückenkonzept für ein problemorientiertes Denken und Arbeiten gesehen werden (Biermann et al. 2012). Nur auf Basis einer fundierten Auseinandersetzung zwischen den Fächern kann sich eine reiche und vielschichtige Debatte zum Menschenzeitalter entwickeln. In diesem interdisziplinären Dialog sollte sich die Politikwissenschaft aktiv einbringen. Denn ohne politikwissenschaftliche Analysen bleiben Themen wie die Komplexität von Governance, Macht- und Gerechtigkeitsfragen, gesellschaftliche Naturverhältnisse und ihre Gestaltbarkeit im Anthropozän unterbeleuchtet.

\section{Fazit: Mehr Engagement der Politikwissenschaft}

Die naturwissenschaftlichen Erkenntnisse zum wachsenden Eingriff des Menschen in das Erdsystem haben in vielen Teilen der Welt zu der Einsicht geführt, dass eine grundlegende Transformation unserer Lebens- und Wirtschaftsweise notwendig ist, um die Erde als Lebensort für die am stärksten verwundbarsten Gruppen der Gesellschaft und zukünftige Generationen zu erhalten. Dennoch schreiten globale Umweltveränderungen wie der Klimawandel, die Verschmutzung der Ozeane oder das Artensterben, die Bodendegradation und die Zerstörung von natürlichen Stoffkreisläufen sowie viele andere Umweltveränderungen weiter voran. Um die Ursachen dieser Probleme zu verstehen und den notwendigen politischen und gesellschaftlichen Wandel einzuleiten, bedarf es politikwissenschaftlicher Perspektiven und Analysen als zentrale Wegmarken.

Wir haben in diesem Beitrag einige dieser Wegmarken hervorgehoben. Erstens ist entgegen der vorherrschenden Annahme in einigen naturwissenschaftlichen Kreisen kein einfaches Management der komplexen Zusammenhänge des Erdsystems möglich. Vielmehr sind Governance-Ansätze ebenso komplex wie schwierig planbar und immer mit unsicherem Ausgang und nichtintendierten Effekten verbunden. Zweitens kann die Politische Theorie wichtige Denkanstöße geben, damit Visionen und Innovationen für politische und gesellschaftliche Reformen entwickelt werden können. Die Schaffung von Zukunftsgremien auf lokaler, nationaler und globaler Ebene ist ein prominentes Beispiel, das über die klassische Bürgerbeteiligung hinausgeht und die Interessen und Rechte der Jugend und zukünftiger Generationen in Politikprozesse einbezieht (Tremmel 2019). Drittens sind politikwissenschaftliche Einsichten gefragt, um gemeinsam mit anderen Disziplinen auf die verschiedenen globalen Veränderungsprozesse adäquate Antwortstrategien zu formulieren.

Insgesamt muss die aktuelle Debatte über das Anthropozän um wesentliche Aspekte erweitert werden. Dies betrifft neben der rein naturwissenschaftlichen Aus- 
einandersetzung, die es zu überwinden gilt, auch die Haltung der Politikwissenschaft. Das Anthropozän sollte kein Nischenthema für die Umweltpolitikforschung bleiben, noch kann es allein mit den Konzepten und Instrumenten der Politikfeldanalyse verstanden werden. Um die weitreichenden politischen und gesellschaftlichen Implikationen des Anthropozän herauszuarbeiten, bedarf es vielmehr des gesamten theoretischen und thematischen Repertoires unserer Disziplin. Politikwissenschaftliche Perspektiven auf das Anthropozän-Konzept bereichern die Anthropozän-Debatte, indem sie die politische Dimension der sozial-ökologischen Transformation sichtbar machen. Gleichzeitig bieten sie die Möglichkeit, mithilfe des reichen Schatzes der politischen Theorie über die Wirkungs- und Gestaltungskraft der Menschheit zu reflektieren. Daher fordern wir mehr Engagement der Politikwissenschaft in der interdisziplinären Anthropozän-Debatte, um Bausteine für die notwendige Nachhaltigkeitstransformation zu liefern.

Funding Open Access funding enabled and organized by Projekt DEAL.

Open Access Dieser Artikel wird unter der Creative Commons Namensnennung 4.0 International Lizenz veröffentlicht, welche die Nutzung, Vervielfältigung, Bearbeitung, Verbreitung und Wiedergabe in jeglichem Medium und Format erlaubt, sofern Sie den/die ursprünglichen Autor(en) und die Quelle ordnungsgemäß nennen, einen Link zur Creative Commons Lizenz beifügen und angeben, ob Änderungen vorgenommen wurden.

Die in diesem Artikel enthaltenen Bilder und sonstiges Drittmaterial unterliegen ebenfalls der genannten Creative Commons Lizenz, sofern sich aus der Abbildungslegende nichts anderes ergibt. Sofern das betreffende Material nicht unter der genannten Creative Commons Lizenz steht und die betreffende Handlung nicht nach gesetzlichen Vorschriften erlaubt ist, ist für die oben aufgeführten Weiterverwendungen des Materials die Einwilligung des jeweiligen Rechteinhabers einzuholen.

Weitere Details zur Lizenz entnehmen Sie bitte der Lizenzinformation auf http://creativecommons.org/ licenses/by/4.0/deed.de.

\section{Literatur}

Alcaraz, Jose M., Katherine Sugars, Katerina Nicolopoulou, und Francisco Tirado. 2016. Cosmopolitanism or globalization: the Anthropocene turn. Society and Business Review 11(3):313-332.

Bennett, Elena M., Martin Solan, Reinette Biggs, Timon McPhearson, Albert V. Norström, Per Olsson, Laura Pereira, Garry D. Peterson, Ciara Raudsepp-Hearne, und Frank Biermann. 2016. Bright spots: seeds of a good anthropocene. Frontiers in Ecology and the Environment 14(8):441-448.

Berkes, Fikret. 2017. Environmental governance for the Anthropocene? Social-ecological systems, resilience, and collaborative learning. Sustainability 9(7):1-12.

Biermann, Frank. 2014. Earth system governance: world politics in the Anthropocene. Earth system governance : a core research project of the international human dimensions programme on global environmental change. Cambridge: MIT Press.

Biermann, Frank, und Eva Lövbrand. 2019. Anthropocene encounters: new directions in green political thinking. Cambridge: Cambridge University Press.

Biermann, Frank, Kenneth Abbott, Steinar Andresen, Karin Bäckstrand, Steven Bernstein, Michele M. Betsill, Harriet Bulkeley, et al, 2012. Navigating the Anthropocene: improving earth system governance. Science 335(6074):1306-1307. https://doi.org/10.1126/science.1217255.

Biermann, Frank, Philipp Pattberg, Harro van Asselt, und Fariborz Zelli. 2009. The fragmentation of global governance architectures: a framework for analysis. Global Environmental Politics 9(4):14-40.

Blühdorn, Ingolfur. 2020. The legitimation crisis of democracy: emancipatory politics, the environmental state and the glass ceiling to socio-ecological transformation. Environmental Politics 29(1):38-57. 
Bosselmann, Klaus. 2015. Earth governance: trusteeship of the global commons. Cheltenham: Edward Elgar.

Chandler, David, und Julian Reid. 2016. The neoliberal subject: resilience, adaptation and vulnerability. London: Rowman \& Littlefield.

Crutzen, Paul J., und Eugene F. Stoermer. 2000. The "Anthropocene". Global Change Newsletter 41:17-18.

Crutzen, Paul J., und Christian Schwägerl. 2011. Living in the Anthropocene: toward a new global ethos. Yale environment 360. http://e360.yale.edu/feature/living_in_the_anthropocene_toward_a_new_ global_ethos/2363. Zugegriffen: 3. Apr. 2017.

Dobson, Andrew. 2007. Green political thought, 4. Aufl., London: Routledge.

Dryzek, John S., und Jonathan Pickering. 2018. The Politics of the Anthropocene. Oxford: Oxford University Press.

Eckersley, Robyn. 2015. Anthropocene raises risks of earth without democracy and without us. https:// theconversation.com/anthropocene-raises-risks-of-earth-without-democracy-and-without-us-38911U. Zugegriffen: 31. März 2017.

Frodeman, Robert, Julie Thompson Klein, und Roberto Carlos Santos Dos Pacheco. 2017. The oxford handbook of interdisciplinarity. Oxford: Oxford University Press.

González-Ricoy, Iñigo, und Axel Gosseries. 2016. Institutions for future generations. Oxford: Oxford University Press.

Görg, Christoph. 2016. Zwischen Tagesgeschäft und Erdgeschichte: Die unterschiedlichen Zeitskalen in der Debatte um das Anthropozän. GAIA-Ecological Perspectives for Science and Society 25(1):9-13.

Hickmann, Thomas, Lena Partzsch, Philipp Pattberg, und Sabine Weiland. 2019. The Anthropocene debate and political science. New York: Routledge.

Hoffmann, Matthew. 2011. Climate governance at the crossroads: experimenting with a global response after Kyoto. Oxford: Oxford University Press.

Jamieson, Dale W., und Marcello Di Paola. 2016. Political theory for the Anthropocene. In Global political theory, Hrsg. David Held, Maffettone Pietro, 254-280. Cambridge: Polity Press.

Kalfagianni, Agni, und Sophia Skordili. 2018. Localizing global food: short food supply chains as responses to agri-food system challenges. London: Routledge.

Keohane, Robert O., und David G. Victor. 2011. The regime complex for climate change. Perspectives on Politics 9(1):7-23.

Kivimaa, Paula, Mikael Hildén, Dave Huitema, Andrew Jordan, und Jens Newig. 2017. Experiments in climate governance: a systematic review of research on energy and built environment transitions. Journal of Cleaner Production 169:17-29.

Langmuir, Charles H., und Wally Broecker. 2012. How to build a habitable planet: the story of earth from the big bang to humankind. Princeton: Princeton University Press.

Lasswell, Harold. 1936. Politics: who gets what, when, how. New York: McGraw Hall.

Lenton, Timothy M. 2011. Early warning of climate tipping points. Nature Climate Change 1(4):201-209.

Lövbrand, Eva, Silke Beck, Jason Chilvers, Tim Forsyth, Johan Hedrén, Mike Hulme, Rolf Lidskog, und Eleftheria Vasileiadou. 2015. Who speaks for the future of earth? How critical social science can extend the conversation on the Anthropocene. Global Environmental Change 32:211-218.

Lövbrand, Eva, Johannes Stripple, und Bo Wiman. 2009. Earth system governmentality: reflections on science in the Anthropocene. Global Environmental Change 19(1):7-13.

Manemann, Jürgen. 2014. Kritik des Anthropozäns: Plädoyer für eine neue Humanökologie. Bielefeld: transcript.

McKibben, Bill. 1990. Das Ende der Natur. München: List.

Okereke, Chukwumerije. 2010. Climate justice and the international regime. Wiley interdisciplinary reviews: climate change 1(3):462-474.

Orsini, Amandine, Philippe Le Prestre, Peter M. Haas, Malte Brosig, Philipp Pattberg, Oscar Widerberg, Laura Gomez-Mera, Neil E.Harrison Jean-Frédéric Morin, und Robert Geyer. 2019. Complex systems and international governance. International Studies Review. https://doi.org/10.1093/isr/viz020

Overdevest, Christine, und Jonathan Zeitlin. 2014. Assembling an experimentalist regime: transnational governance interactions in the forest sector. Regulation \& Governance 8(1):22-48.

Pattberg, Philipp, und Michael Davies-Venn. 2020. Dating the Anthropocene. In The Anthropocenic turn, Hrsg. Gabriele Dürbeck, Philip Hüpkes, 130-149. New York: Routledge.

Pattberg, Philipp, und Oscar Widerberg. 2019. Smart mixes and the challenge of complexity: the example of global climate governance. In Smart mixes in relation to transboundary harm: interactions between international, state, and private regulation, Hrsg. Jeroen van Erp, M. Faure, A. Nollkaemper, und N. Philipsen. Cambridge: Cambridge University Press. 
Pattberg, Philipp, und Fariborz Zelli. 2016. Environmental politics and governance in the Anthropocene: institutions and legitimacy in a complex world. London: Routledge.

Reid, Walter V., Deliang Chen, L. Goldfarb, Heide Y.T. Hackmann Lee, K. Mokhele, Elinor Ostrom, et al, 2010. Earth system science for global sustainability: grand challenges. Science 330(6006):916-917. https://doi.org/10.1126/science.1196263.

Rockström, Johan, Hans Joachim Schellnhuber, Brian Hoskins, Veerabhadran Ramanathan, Peter Schlosser, Guy Pierre Brasseur, Owen Gaffney, Carlos Nobre, Malte Meinshausen, und Joeri Rogelj. 2016. The world's biggest gamble. Earth's Future 4(10):465-470.

Rothe, Delf. 2020. Governing the end times? Planet politics and the secular eschatology of the Anthropocene. Millennium: Journal of International Studies 48(2):143-164.

Schmidt, Jeremy J., Peter G. Brown, und Christopher J. Orr. 2016. Ethics in the Anthropocene: a research agenda. The Anthropocene Review 3(3):188-200.

Steffen, Will, Åsa Persson, Lisa Deutsch, Jan Zalasiewicz, Mark Williams, Katherine Richardson, Carole Crumley, Paul Crutzen, Carl Folke, und Line Gordon. 2011. The Anthropocene: from global change to planetary stewardship. Ambio: A Journal of the Human Environment 40(7):739-761.

Steffen, Will, Johan Rockström, Katherine Richardson, Timothy M. Lenton, Carl Folke, Diana Liverman, Colin P. Summerhayes, Anthony D. Barnosky, Sarah E. Cornell, und Michel Crucifix. 2018. Trajectories of the earth system in the Anthropocene. Proceedings of the National Academy of Sciences of the United States of America 115(33):8252-8259.

Stirling, Andrew. 2014. Emancipating transformations: from controlling 'the transistion' to culturing plural radical progress. In STEPS Working Paper. Sussex: University of Sussex.

Tremmel, Jörg. 2015. Parliaments and future generations. In The politics of sustainability: philosophical perspectives, Hrsg. Dieter Birnbacher, May Thorseth, 212-233. London: Routledge.

Tremmel, Jörg. 2019. The Anthropocene concept as a wake-up call for reforming democracy. In The Anthropocene debate and political science, Hrsg. Thomas Hickmann, Lena Partzsch, Philipp Pattberg, und Sabine Weiland, 219-236. New York: Routledge.

Vaughan, Naomi E., und Timothy M. Lenton. 2011. A review of climate Geoengineering proposals. Climatic Change 109(3-4):745-790. 